\title{
Data-driven and learning-based interpolations of along-track Nadir and wide-swath SWOT altimetry observations
}

\author{
Beauchamp Maxime ${ }^{1,{ }^{*}}$, Fablet Ronan ${ }^{1}$, Ubelmann Clement ${ }^{2}$, Ballarotta Maxime ${ }^{3}$, Chapron Bertrand ${ }^{4}$ \\ 1 IMT Atlantique Bretagne Pays Loire, Brest, France. \\ 2 Ocean Next, Grenoble, France. \\ ${ }^{3}$ Collecte Localisat Satellites CLS, Ramonville St Agne, France. \\ ${ }^{4}$ IFREMER, Plouzane, France. \\ * Corresponding author : Maxime Beauchamp, email address : maxime.beauchamp@imt-atlantique.fr
}

\begin{abstract}
:
Over the last years, a very active field of research aims at exploring new data-driven and learning-based methodologies to propose computationally efficient strategies able to benefit from the large amount of observational remote sensing and numerical simulations for the reconstruction, interpolation and prediction of high-resolution derived products of geophysical fields. In this paper, we investigate how they might help to solve for the oversmoothing of the state-of-the-art optimal interpolation $(\mathrm{OI})$ techniques in the reconstruction of sea surface height (SSH) spatio-temporal fields. We focus on a small region, part of the GULFSTREAM and mainly driven by energetic mesoscale dynamics. Based on an Observation System Simulation Experiment (OSSE), we will use the NATL60 high resolution deterministic ocean simulation of the North Atlantic to generate two types of pseudo altimetric observational dataset: alongtrack nadir data for the current capabilities of the observation system and wide-swath SWOT data in the context of the upcoming SWOT mission. We briefly introduce the analog data assimilation (AnDA), an upto-date version of the DINEOF algorithm, and a new NN-based end-to-end learning framework for the representation of spatio-temporal irregulary-sampled data. We evaluate how some of these methods are a significant improvements, particularly by catching up the small scales ranging up to $30-40 \mathrm{~km}$, inaccessible by the conventional methods so far. A clear gain is also demonstrated when assimilating jointly wide-swath SWOT and (agreggated) along-track nadir observations.
\end{abstract}




\section{INTRODUCTION}

Thanks to the ocean surface remote sensing data acquired by different altimetric missions
(TOPEX/Poseidon, ERS-1, ERS-2, Geosat FollowOn, Jason-1, Envisat and OSTM/Jason-2), our understanding of the ocean circulation has been considerably improved over the last decades. But currently, the range of scales over $150 \mathrm{~km}$ remains inaccessible to altimetric derived products because of the limited number of altimetric missions and their spatio-temporal sampling. In this context, a very active field of research now consists in taking advantage of the big amount of data and numerical simulations available to overcome these limits of conventional altimetric products, which motivate complementary developments combining high resolution remote sensing and numerical simulations.

Over the last years, purely data-driven and artifical intelligence (AI)-based algorithms have just been proposed [1], [2], [3], [4], [5] to deal with problems directly related to data assimilation and operational oceanography. More specifically, promising preliminary results have been seen for the sea surface reconstruction and prediction from partial and noisy satellite observations.

In this paper, we propose an intercomparison exercise of several data-driven and learning-based approaches to help for the reconstruction of altimetric fields. As a baseline the DUACS operational processing tool based on well established optimal interpolation (OI) techniques will be considered. In Section II, we present the case study and its dataset, developed within the BOOST-SWOT project framework: the NATL60 high resolution deterministic ocean simulation of the North Atlantic [6]) is used as reference to simulate Sea Surface Height (SSH) along-track observations collected by four nadir, which is typically representative of the current observational altimetric capabilities. As an additional feature for the upcoming 2021 SWOT mission, pseudo-SWOT wide-swath observations also following realistic orbits are generated based on the NATL60 simulation. In Section III, we present the 
data-driven approaches used in the intercomparison: 1) AnDA, a purely data-driven data assimilation scheme combining a patch-based analog forecasting operator with Kalman-based ensemble data assimilation, 2) VE-DINEOF, an EOF-based iterative method to interpolate in space and time the missing data, and 3) learning-based innovative end-to-end learning techniques that aims to learn jointly the Neural Network (NN) representation of the dynamics coupled with a NN-based solver of the targeted minimization problem. Last, in Section IV, we give a detailed evaluation of the results obtained over a small region, part of the GULFSTREAM and mainly driven by energetic mesoscale dynamics.

\section{CASE STUDY AND DATA}

\section{A. NATL60}

The Nature Run (NR) used in this work corresponds to the NATL60 configuration [6] of the NEMO (Nucleus for European Modeling of the Ocean) model. It is one of the most advanced state-of-the-art basinscale high-resolution $\left(1 / 60^{\circ}\right)$ simulation available today, whose surface field effective resolution is about $7 \mathrm{~km}$.

In this work, a specific domain of $10^{\circ} \times 10^{\circ}$, part of the Gulf Stream, is selected (Figure 1) to assess the performance of the data-driven interpolation methods. Over this region, the Sea Surface Height ( $\mathrm{SSH}$ ) is mainly driven by energetic mesoscale dynamics: the resolution of the nature run is thus downgraded to $1 / 20^{\circ}$, which is enough to capture this dynamical regime, while avoiding unnecessary heavy computational time. The NATL60 nature run will then be used as the reference Ground Truth (GT) in an observing system simulation experiments (OSSE). The pseudo-altimetric nadir and SWOT observational datasets will be generated by a realistic sub-sampling of satellite constellations.

\section{B. Nadir}

To provide the pseudo-nadir dataset, supposed to be representative of what is a current pre-SWOT observational altimetric dataset, the groundtracks of 4 altimetric missions (TOPEX/Poseidon, Geosat, Jason-1 and Envisat) picked up from the 2003 constellation, are used to interpolate the NATL60 simulation from October 1st, 2012 to September 29th, 2013, thus covering a whole year of data. A Gaussian white noise with variance $\sigma^{2}=30 \mathrm{~cm}$ is then added to the interpolated NATL60 simulation on the nadir coordinates to simulate the instrumental acquisition noise [7]. Because the spacetime interpolations will focus on a daily-basis temporal resolution, we also build nadir pseudo-observations with an additional strategy by accumulating observations over a time window $t_{k} \pm d$ days centered at time $t_{k}$ in order to increase the daily nadir spatial sampling. As in [4], we investigate the response of the different interpolation techniques when parameter $d$ is either set to 0 or 5 , see Figures $2 \mathrm{a}$ and $2 \mathrm{c}$.

\section{SWOT}

In the same line, SWOT-like pseudo observations are also produced by the swotsimulator tool [8] in its swath mode with an along-track and across-track $2 \mathrm{~km}$ spatial resolution, the same theoretical resolution the upcoming SWOT mission derived products should be able to provide. The nadir mode of the generator also provide pseudo-nadir along track observations though they are not used here. The simulator also adds instrumental noise on the idealized pseudo-SWOT dataset [9], [10]. This noise potentially exhibit strong spacetime correlations. Thus, the pseudo-SWOT observations are first preprocessed (citation++) to filter out these correlated components and avoid major issues in the assimilation and/or learning process of the interpolation methods.

Let precise that over this low-latitude GULFSTREAM domain, the SWOT sampling is irregular leading to sequences of several days with only pseudo-nadir observations.

\section{DUACS OI products}

The DUACS system is an operational production of sea level products for the Marine (CMEMS) and Climate (C3S) services of the E.U. Copernicus program, on behalf of the CNES french space agency. It is mainly based on optimal interpolation techniques whose parameters are fully described in [11]. This methodology has been applied on the previously introduced pseudo along-track nadir and wide-swath SWOT data to generate regular $\left(0.25^{\circ} \mathrm{x} 0.25^{\circ}\right)$ daily gridded maps.

\section{Methods}

The data-driven methods we are investigating aims at solving smaller scales than operational OI products, more adapted to estimate large scale dynamics. Along this line, we are using in the following a multiscale decomposition:

$$
\mathbf{x}=\overline{\mathbf{x}}+d \mathbf{x}+\epsilon
$$

and all the interpolations methods used here will work on the anomaly field $d \mathbf{x}$, seen as the difference between 


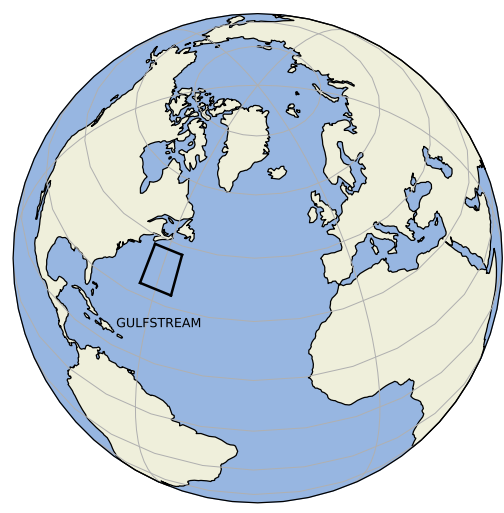

Fig. 1: GULFSTREAM domain

the original field $\mathrm{x}$ and the large scales components provided by the OI. In the end, we hope the effective resolution estimated for the anomaly field $d \mathbf{x}$ to be better than the OI-based representation of the dynamics. In what follows, $\mathbf{y}$ denotes the observational data corresponding to subdomain $\Omega \subset \mathcal{D}, \bar{\Omega}$ denotes the gappy part of the SSH field and index $k$ refers to time $t_{k}$.

\section{A. $A n D A$}

The Analog Data Assimilation (AnDA) is a purely data-driven data assimilation method, that introduces a statistical operator $\mathcal{A}$ as a substitute for the dynamical model $\mathcal{M}$, leading to the following state-space formulation :

$$
\begin{cases}d \mathbf{x}_{k+1} & =\mathcal{A}_{k+1}\left(d \mathbf{x}_{k}\right)+\boldsymbol{\mu}_{k} \\ d \mathbf{y}_{k} & =\mathcal{H}_{k}\left(d \mathbf{x}_{k}\right)+\varepsilon_{k}\end{cases}
$$

The analog forecasting operator $\mathcal{A}: \mathbf{d x}_{k-1}^{a} \mapsto \mathbf{d x}_{k}^{f}$ , where superscripts $a$ and $f$ respectively relies to analysis and forecast, is built from the $K$ most similar states to $\mathbf{d x}_{k-1}^{a}$ in the available past state dynamics catalog, supposed to be large enough to describe the space-time evolution of the processes. More precisely, $\mathbf{d x}_{k}^{f}$ is sampled from the Gaussian prior $\mathbf{d x}_{k}^{f} \mid \mathbf{d} \mathbf{x}_{k-1}^{a} \sim$ $\mathcal{N}\left(\boldsymbol{\mu}_{k}, \boldsymbol{\Sigma}_{k}\right)$, where the mean $\boldsymbol{\mu}_{k}$ and the covariance matrix $\Sigma_{k}$ are estimated using the so-called locally linear model [1], i.e. a weighted linear regression between the $K$ nearest analogs and their successors.

As in [4], a patch-based version of AnDA coupled with an EOF-based representation of the individual patches is used. The anomaly field $d \mathbf{x}$ is splitted into 169 vectorized patches $\mathbf{p}(\mathbf{s}, t)$ of sizes $1^{\circ} \times 1^{\circ}$, corresponding to 20 pixels $\times 20$ pixels, with overlapping areas of 5 pixels. An EOF-based decomposition of each individual vectorized anomaly patches is then carried out to deal with the curse of dimensionality. Finally, the whole AnDA algorithm is performed at the patch-level, meaning that both the analog prediction and the assimilation are done onto the lower-dimensional space of their EOF-based representation. A final post-processing step (denoted as post-AnDA) is used to project the prediction onto the original space-time domain and average the overlapping patches to smooth out some blocky artefacts coming from the patch decomposition. On this last point, an improvement can be considered by using a convolutional neural network (CNN) to learn how to reconstruct the whole domain from the set of overlapping patches, as in [5].

\section{B. VE-DINEOF}

VE-DINEOF is a state-of-the-art interpolation approach [12] using an EOF-based iterative filling strategy. Typically the large-scale component provided by the OI is used (or 0 values if working on the anomaly) as a first guess to fill in the missing data over $\Omega$. After each iteration and until convergence, the field is projected onto the $N$ most significant EOF components of the lower dimensional space and new values for the missing data are used based on the updated reconstruction of the field. Finally, the VE-DINEOF algorithm is here proposed in its patch-based version, in the exact similar setting proposed for AnDA.

\section{End-to-end NN-learning}

An end-to-end learning representation has recently been introduced in [13] to deal with image sequences involving potentially large missing data rates. In this framework, an energy-based representation $U_{\theta}$ to minimize is introduced :

$$
U_{\theta}(d \mathbf{x})=\|d \mathbf{x}-\psi(d \mathbf{x})\|^{2}
$$

where the operator $\psi$ denotes a NN-based representation of the underlying processes. Typically, two NNbased energy parametrizations are considered. First, a classic convolutional auto-encoders (ConvAE) representations $\psi(\cdot)=\phi_{D}\left(\phi_{E}(\cdot)\right)$ where the encoding operator $\phi_{E}$ maps the anomaly state $d \mathbf{x}$ onto a lowerdimensional space and the decoder $\phi_{D}$ has to project this encoded representation in the original space. Second, NN-based Gibbs-Energy (GENN) representations where $d \mathbf{x}_{s}$, the anomaly observed at location $\mathbf{s} \in \mathcal{D}$, is supposed to be explained by the potential function $\psi\left(d \mathbf{x}_{\delta s}\right)$ with $\delta s$ a predefined neighbourhood of site 


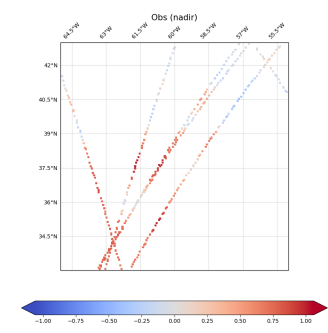

(a) nadir $(d=0)$

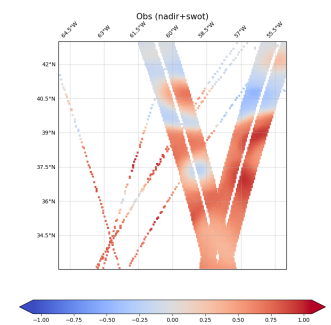

(b) nadir $(d=0)+\operatorname{swot}$

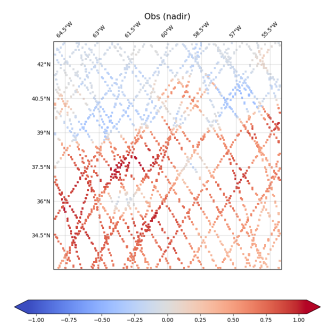

(c) nadir $(d=5)$

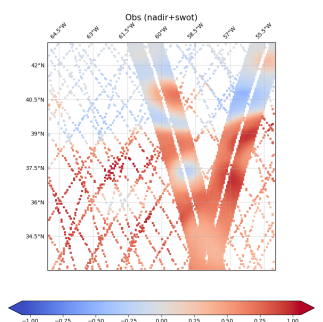

(d) nadir $(d=5)+$ swot

Fig. 2: 0 and 5-days accumulated along-track nadir and wide-swath pseudo-observations for August 4, 2013

$\mathbf{s}$, thus relating this representation to Markovian priors embedded in CNNs. A low energy-state $U_{\theta}(d \mathbf{x})=$ $\int_{\mathcal{D}} U_{\theta}\left(d \mathbf{x}_{s}\right) d s$ over the entire domain $\mathcal{D}$ ensures to provide a good state space reconstruction. Based on this NN-parametrization of operator $\psi$, a fixed-point algorithm (FP) is used during the training for solving the minimization of the reconstruction error for the observed error. It is parameter-free and easily implemented as a $\mathrm{NN}$ in a joint solution with the $\mathrm{NN}$ parametrization of $U_{\theta}$ for the interpolation problem. Let precise that during the learning phase, anomaly image time series $d \mathbf{x}_{k \pm d T}=d \mathbf{x}_{k-d T: k+d T}$ are built with time window $d T=5$, centered on time $t_{k}$.

The two $\mathrm{NN}$-architectures are then refered as $\mathrm{FP}$ ConvAE and FP-GENN. In [13], an additional NNbased reinterpretation of an iterative gradient-based descent solution is proposed to solve the issue of optimizing the parameters $\theta$ in energy $U_{\theta}$.

\section{Evaluation}

A specific aspect of this work consists in the period of data available because the NATL60 native run is only one-year long which is relatively short in comparison with the training period typically used in the previous related work mentioned in Introduction. To get around this issue, we decide to build four 20-days long validation period homogeneously distributed along this one-year dataset (see the starting dates reported on Figures 3), supposed to be representative of the different seasonality effects that may be encountered during the year. We first have to discuss the time window parameter $d$ related to the aggregation of alongtrack data over a specific day $t_{k}$, see Section II.B. A same value of this parameter may not be optimal for all the interpolators: AnDA exhibits a better performance when considering only along-track nadir data of the day $(d=0)$, thus contradicting the previous optimal results of $d=5$ found by [4] over the Mediterranean sea, which may indicate AnDA responds differently to the along-track aggregation strategy depending on the energetic dynamical regime of the region. On the other hand, both FP-ConvAE and FP-GENN interpolators performs better (not shown here) by aggregating nadir data over a 5-day time window. As a consequence, the results presented in what follows will use value of $d=0$ for AnDA and VE-DINEOF and $d=5$ for FP-ConvAE and FP-GENN. Next, to evaluate the behaviour of the different interpolators on both alongtrack nadir samplings and their fusion with wide-swath SWOT datasets and make the comparison possible, we have to preliminary define which configuration the NN-based interpolators will be used. Here, a fully unsupervised version of FP-GENN is used in which both the input and target are only made of the pseudoobservations. The DUACS OI product is also used as a covariate in the inputs, because we think that this may give a prior information about how the anomaly field $d \mathbf{x}$ is distributed. Regarding FP-ConvAE, because it generally shows lower performance, probably because auto-encoders may not be relevant for the reconstruction of fine-scale processes, it will be used in the following as a low-rated NN-scheme among the NN-based interpolators in its mid-supervised configuration: the inputs are the pseudo-observations and the gap-free NATL60 outputs are used as targets. Figure 3 presents the daily nRMSE of the different interpolators: it can be seen how FP-GENN significantly outperforms the conventional OI-based interpolator, but also the other data-driven algorithms used in the experiment. AnDA still remains quite efficient at the very beginning of the four 20-days validation period, which is probably related to a strong persistence of the mesoscale dynamics of the SSH over the region. In other words, the oneyear catalog (minus the 80 validation days) obviously 
enable to build a good analog forecasting operator when knowing the short-term dynamics, but its accuracy quickly decays afterwards, which may not be fair for AnDA that probably requires longer simulations-based catalog in this low-latitude GULFSTREAM region with large Rossby radius of deformation.

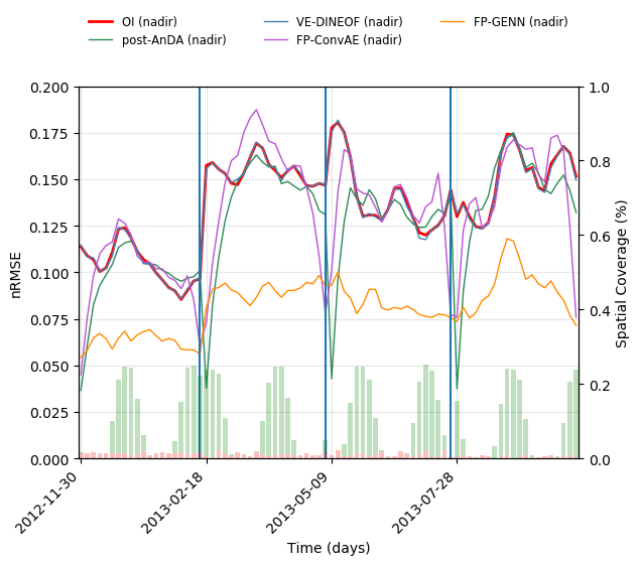

(a) nadir

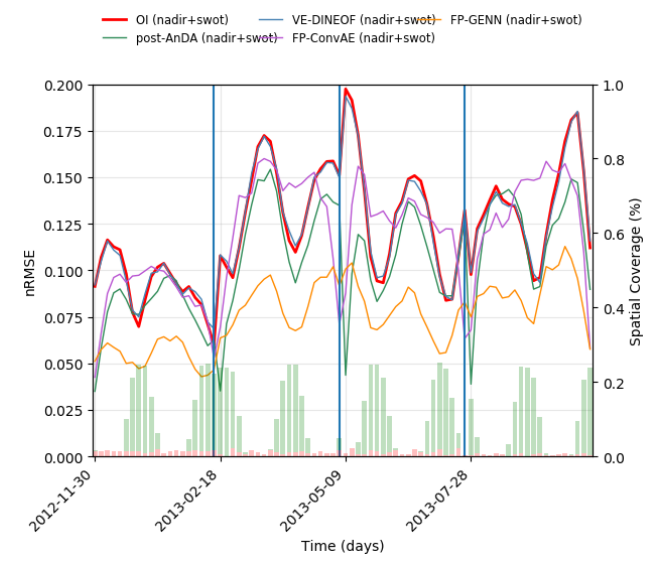

(b) nadir+swot

Fig. 3: Daily spatial nRMSE computed on the 80-days non-continuous validation period for OI, (post-)AnDA, VE-DINEOF, FP-ConvAE and FP-GENN. The spatial coverage of 0-days accumulated along-track nadir and wide-swath SWOT data are respectively provided by the red and green-colored barplots

The Taylor diagram in Figure 4, here calculated over the 80 validation days and focusing only on small-scale structures by applying a high-pass filter that spectrally separates the horizontal scales ranging in the order of $150 \mathrm{~km}$, also confirms our first findings.

In Table I, we provide some statistics (median, 5th and 95th percentile) about the daily spatial SSH and gradient nRMSE, computed using the $4 \times 10$ days at the center of the 4 validation periods in order to avoid some SSH persistency-related overrated performances. FP-
GENN clearly stands out from the other interpolators, which motivate its future use for irregularly-sampled data with large missing data rates. In addition, because its reconstruction scores (where pseudo-observations are available) still remain overall satisfactory, in particular when considering the joint learning on nadir and SWOT data, these results are supplementary arguments on account of this markovian NN-based formulation.

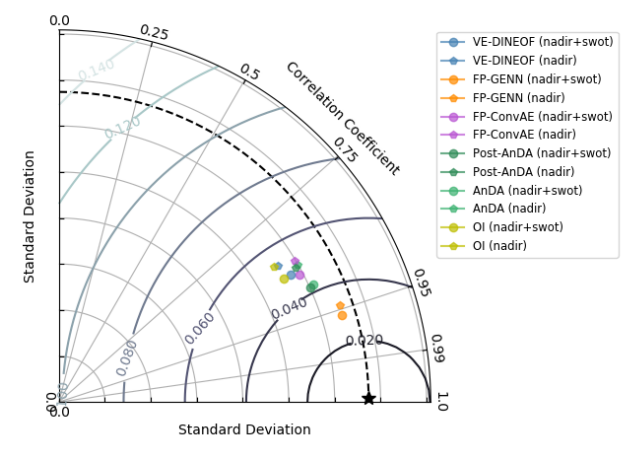

Fig. 4: Taylor diagram computed on the 80-days noncontinuous validation period for OI, (post-)AnDA, VEDINEOF, FP-ConvAE and FP-GENN

Last, when computing the radially averaged power spectra as a spatial domain averaged over the 80-days validation period and the associated signal-to-noise ratio for joint use of along-track nadir with SWOT data (not shown here), we observe that AnDA and FP-GENN lead to a better constraint of the SSH spectrum compared to the actual OI capabilities. In particular, these two methods produce a spectrum closer to the ground truth real spectrum, by catching up the submesoscale range up to $50 \mathrm{~km}$ if along-track nadir are only used, a result improved up to $40 \mathrm{~km}$ for AnDA and FP-GENN when considering additional SWOT data. Let note the importance of the patch-based AnDA post-processing on its performance: its overestimation by the blocky patch-based AnDA rough outputs is partly mitigated thanks to the smoothing produced by averaging the patches overlapping areas. This result may certainly be further improved, for instance by training a CNN rather than using a simple average-based smoothing. To further enhance the vizualisation of the improvements brought by the different interpolators, Figure 5 and Figure 6 depict the SSH and its gradient (module) reference ground truth as well as the global reconstruction obtained with the OI, (post-)AnDA, VE-DINEOF, FP-ConvAE and FP-GENN when using along-track nadir only on August 4, 2013. Figures 7 and Figure 8 illustrate the same interpolations but considering additional wide-swath pseudo-observations. To support 


\begin{tabular}{|c|c|c|c|c|}
\hline & Model type & median & $q_{0.5}$ & $q_{0.95}$ \\
\hline \multirow{5}{*}{$\stackrel{\Xi}{\overparen{Z}}$} & OI & 0.14 & 0.10 & 0.17 \\
\hline & AnDA & 0.14 & 0.10 & 0.17 \\
\hline & VE-DINEOF & 0.14 & 0.10 & 0.17 \\
\hline & FP-ConvAE & 0.14 & 0.10 & 0.18 \\
\hline & FP-GENN & 0.09 & 0.06 & 0.11 \\
\hline \multirow{5}{*}{ 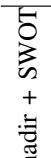 } & OI & 0.12 & 0.08 & 0.17 \\
\hline & AnDA & 0.11 & 0.08 & 0.15 \\
\hline & VE-DINEOF & 0.12 & 0.09 & 0.17 \\
\hline & FP-ConvAE & 0.13 & 0.09 & 0.16 \\
\hline & FP-GENN & 0.08 & 0.05 & 0.10 \\
\hline
\end{tabular}

\begin{tabular}{|c|c|c|c|c|}
\hline & Model type & median & $q_{0.5}$ & $q_{0.95}$ \\
\hline \multirow{5}{*}{$\underset{\Xi}{\exists}$} & $\nabla_{\text {OI }}$ & 0.52 & 0.40 & 0.64 \\
\hline & $\nabla_{\mathrm{AnDA}}$ & 0.52 & 0.41 & 0.62 \\
\hline & $\nabla_{\mathrm{VE}-\mathrm{DINEOF}}$ & 0.53 & 0.41 & 0.65 \\
\hline & $\nabla_{\mathrm{FP}-\mathrm{ConvAE}}$ & 0.56 & 0.43 & 0.70 \\
\hline & $\nabla_{\mathrm{FP}-\mathrm{GENN}}$ & 0.41 & $\mathbf{0 . 3 3}$ & 0.48 \\
\hline \multirow{5}{*}{$\begin{array}{l}5 \\
0 \\
\text { s } \\
+ \\
+ \\
= \\
0\end{array}$} & $\nabla_{\text {OI }}$ & 0.49 & 0.35 & 0.66 \\
\hline & $\nabla_{\mathrm{AnDA}}$ & 0.45 & 0.33 & 0.61 \\
\hline & $\nabla_{\mathrm{VE}-\mathrm{DINEOF}}$ & 0.52 & 0.40 & 0.67 \\
\hline & $\nabla_{\mathrm{FP}-\mathrm{ConvAE}}$ & 0.54 & 0.41 & 0.65 \\
\hline & $\nabla_{\mathrm{FP}-\mathrm{GENN}}$ & 0.38 & 0.30 & 0.45 \\
\hline
\end{tabular}

TABLE I: Daily SSH and SSH gradient field median nRMSE and associated 5th and 95th percentile computed using the $4 \times 10$ days at the center of the 4 validation periods for OI, (post-)AnDA, VE-DINEOF, FP-ConvAE and FP-GENN for both nadir use only and joint assimilation/learning with wide-swath SWOT data

what has already been said through the performance analysis previously discussed, FP-GENN using 5-days accumulated nadir observations appears closer to the ground truth $\mathrm{SSH}$ field than the reconstruction obtained with FP-ConvAE using a similar solver but a simple auto-encoder representation of the dynamics. The latter clearly oversmoothes the true field and also exhibits some unnecessary artefacts on the SSH gradient thus explaining the noisy-related small scale energies on the spectra. The same artefacts appears on the VEDINEOF mapping which exhibits discontinuities between the known wide-swath-informed areas and the filled missing data. Last, AnDA also behaves well, especially because the wide-swath SWOT data coverage on this specific day is important, getting its performance closer to FP-GENN than the day without the 2DSWOT information. Besides methodological aspects, the use of additional 2D wide-swath swot information significantly improves the gradient reconstruction, which is clearly apparent for AnDA and FP-GENN when looking at Figures $6 \mathrm{c} / \mathrm{f}$ and Figures 8c/f.

\section{CONCLUSION}

In this short paper, we described a selection of data-driven and learning-based methodologies to apply on altimetric along-track nadir and wide-swath SWOT datasets for the reconstruction of SSH fields. We quantify the potential gain provided by the upcoming SWOT mission on this approaches and demonstrate the great potential of well-designed NN-based method that gives here the best results and seems to open a future research avenue in data assimilation related issues for the learning of geophysical dynamics.

\section{ACKNOWLEDGMENTS}

Funding for the authors was provided by the National Centre for Space Studies (CNES), the French government space agency.

\section{REFERENCES}

[1] R. Lguensat, P. Tandeo, P. Aillot, and R. Fablet, "The Analog Data Assimilation," Monthly Weather Review, 2017.

[2] R. Lguensat, P. Huynh Viet, M. Sun, G. Chen, T. Fenglin, B. Chapron, and R. Fablet, "Data-driven Interpolation of Sea Level Anomalies using Analog Data Assimilation,” Oct. 2017.

[3] R. Fablet, P. H. Viet, and R. Lguensat, "Data-Driven Models for the Spatio-Temporal Interpolation of Satellite-Derived SST Fields," IEEE Trans. on Computational Imaging, vol. 3, no. 4, pp. 647-657, 2017.

[4] M. Lopez-Radcenco, A. Pascual, L. Gomez-Navarro, A. AissaEl-Bey, B. Chapron, and R. Fablet, "Analog Data Assimilation of Along-Track Nadir and Wide-Swath SWOT Altimetry Observations in the Western Mediterranean Sea," IEEE Journal of Selected Topics in Applied Earth Observations and Remote Sensing, pp. 1-11, 2019.

[5] S. Ouala, R. Fablet, C. Herzet, B. Chapron, A. Pascual, F. Collard, and L. Gaultier, "Neural Network Based Kalman Filters for the Spatio-Temporal Interpolation of SatelliteDerived Sea Surface Temperature," Remote Sensing, vol. 10, no. 12, p. 1864, 2018.

[6] J.-M. Molines, "meom-configurations/NATL60-CJM165: NATL60 code used for CJM165 experiment."

[7] M. Ballarotta, C. Ubelmann, M.-I. Pujol, G. Taburet, F. Fournier, J.-F. Legeais, Y. Faugère, A. Delepoulle, D. Chelton, G. Dibarboure, and N. Picot, "On the resolutions of ocean altimetry maps," Ocean Science, vol. 15, no. 4, pp. 1091-1109, 2019.

[8] L. Gaultier, C. Ubelmann, and L.-L. Fu, "The Challenge of Using Future SWOT Data for Oceanic Field Reconstruction," Journal of Atmospheric and Oceanic Technology, vol. 33, pp. 119-126, Nov. 2015.

[9] D. Esteban-Fernandez, "Swot project mission performance and error budget document," tech. rep., JPL, NASA, 2014.

[10] L. Gaultier and C. Ubelmann, "Swot simulator documentation," tech. rep., JPL, NASA, 2010.

[11] G. Taburet, A. Sanchez-Roman, M. Ballarotta, M.-I. Pujol, J.-F. Legeais, F. Fournier, Y. Faugere, and G. Dibarboure, "DUACS DT2018: 25 years of reprocessed sea level altimetry products," vol. 15, no. 5, pp. 1207-1224. Publisher: Copernicus $\mathrm{GmbH}$.

[12] B. Ping, F. Su, and Y. Meng, "An Improved DINEOF Algorithm for Filling Missing Values in Spatio-Temporal Sea Surface Temperature Data," PLOS ONE, vol. 11, no. 5, p. e0155928, 2016.

[13] R. Fablet, L. Drumetz, and F. Rousseau, "End-to-end learning of optimal interpolators for geophysical dynamics." 2019. 


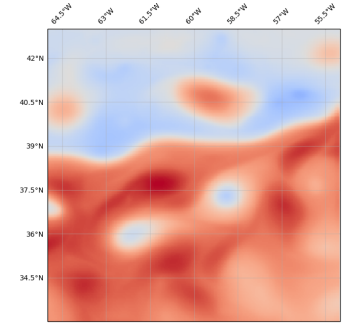

(a) Ground Truth (SSH)

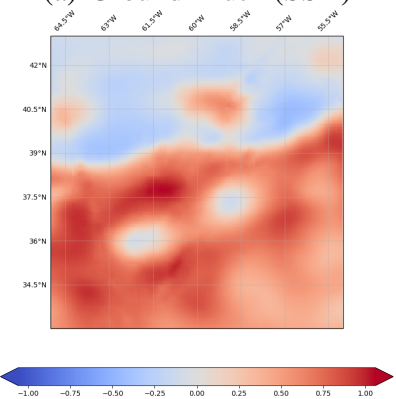

(d) VE-DINEOF

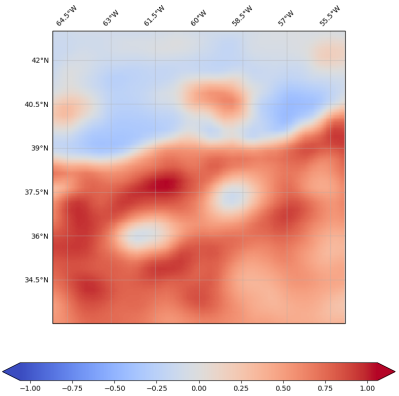

(b) $\mathrm{OI}$

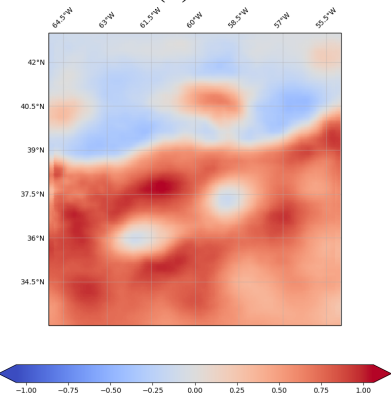

(e) FP-ConvAE

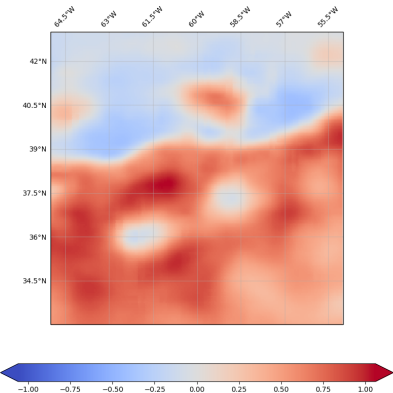

(c) Post-AnDA

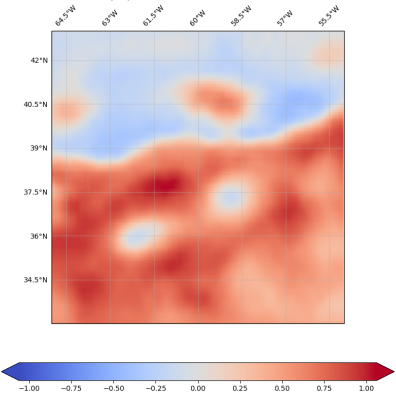

(f) FP-GENN

Fig. 5: Global SSH field reconstruction (August 4, 2013) obtained by OI, (post-)AnDA, VE-DINEOF, FP-ConvAE and FP-GENN using along-track nadir data only

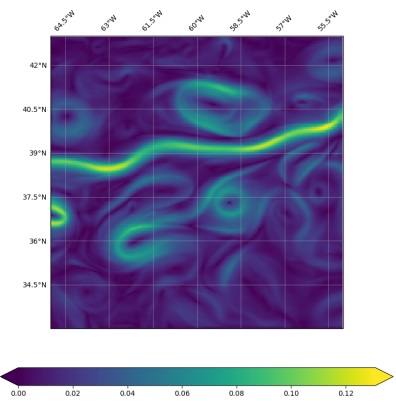

(a) Ground Truth $\left(\nabla_{\mathrm{SSH}}\right)$

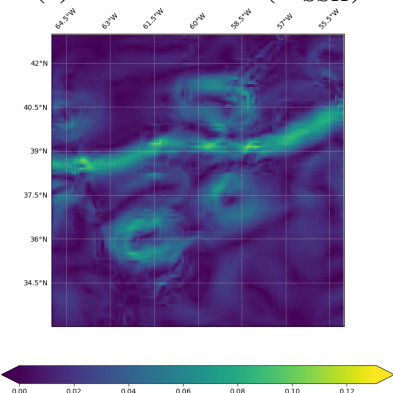

(d) VE-DINEOF

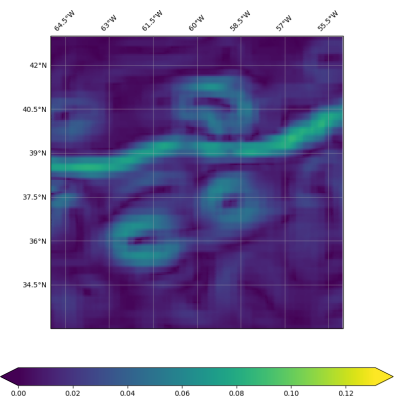

(b) $\mathrm{OI}$

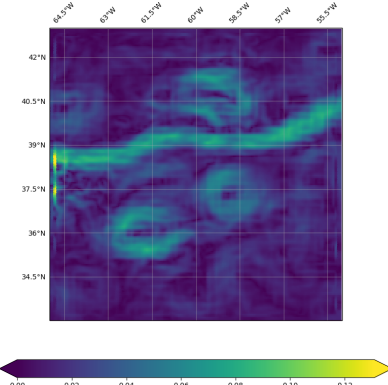

(e) FP-ConvAE

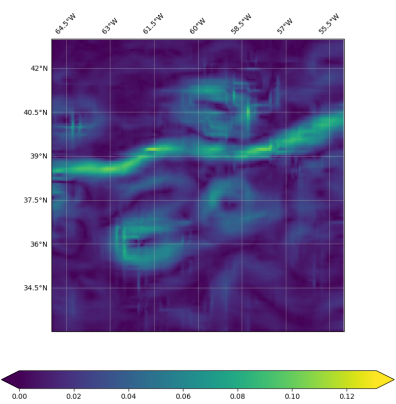

(c) Post-AnDA

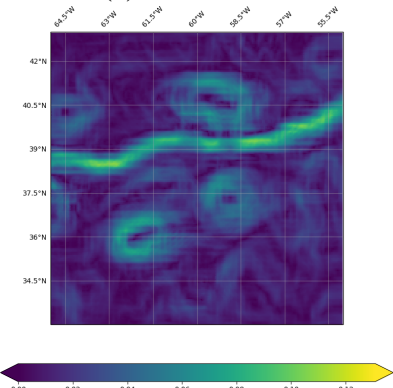

(f) FP-GENN

Fig. 6: Global SSH gradient field reconstruction (August 4, 2013) obtained by OI, (post-)AnDA, VE-DINEOF, FP-ConvAE and FP-GENN using along-track nadir data only 


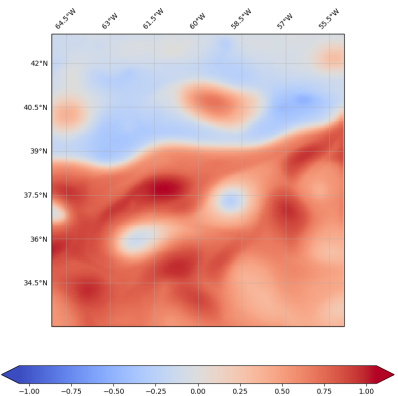

(a) Ground Truth (SSH)

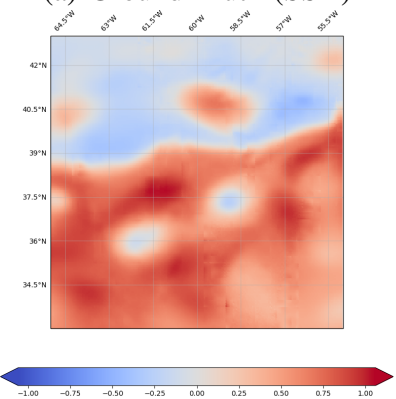

(d) VE-DINEOF

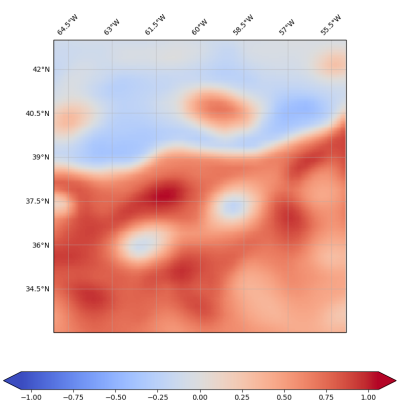

(b) $\mathrm{OI}$

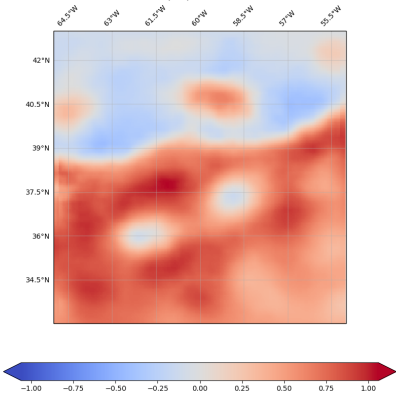

(e) FP-ConvAE

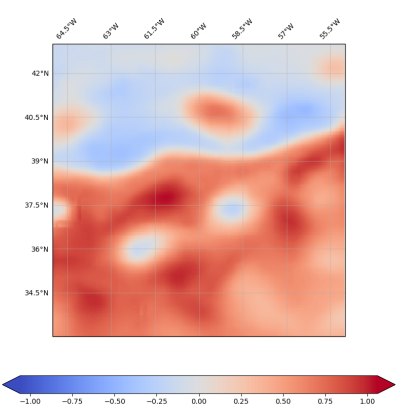

(c) Post-AnDA

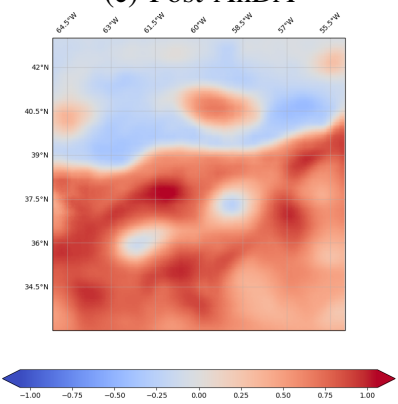

(f) FP-GENN

Fig. 7: Global SSH field reconstruction (August 4, 2013) obtained by OI, (post-)AnDA, VE-DINEOF, FP-ConvAE and FP-GENN for a joint assimilation/learning of along-track nadir with wide-swath SWOT data

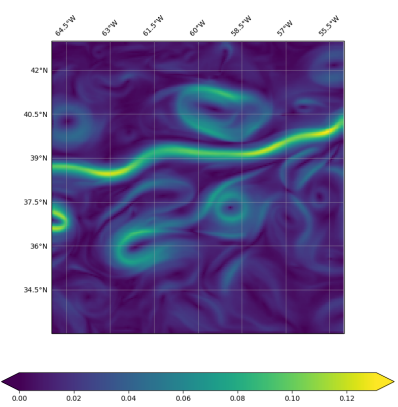

(a) Ground Truth $\left(\nabla_{\mathrm{SSH}}\right)$

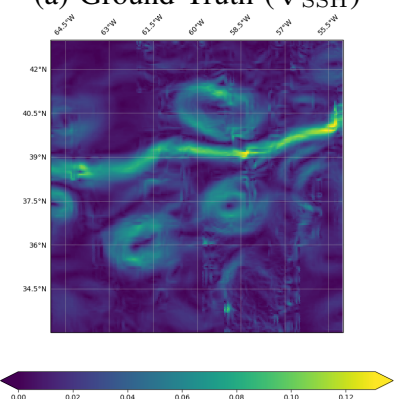

(d) VE-DINEOF

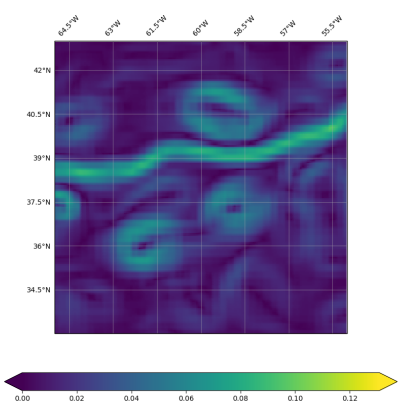

(b) $\mathrm{OI}$

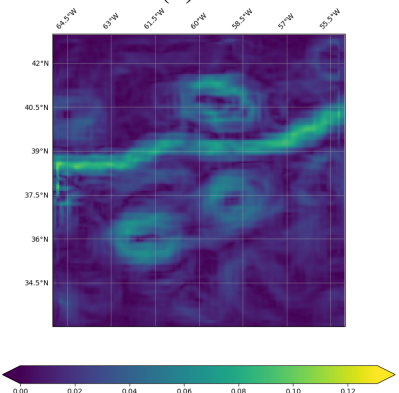

(e) FP-ConvAE

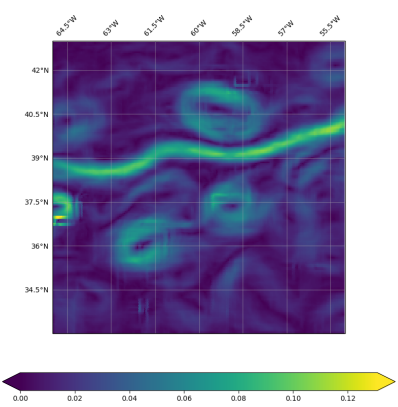

(c) Post-AnDA

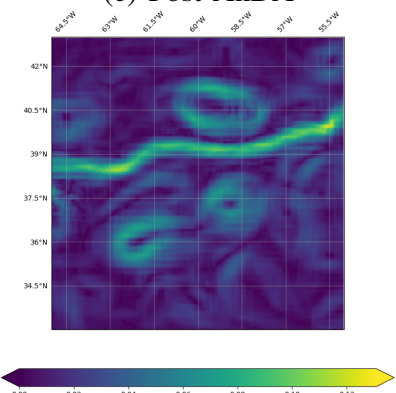

(f) FP-GENN

Fig. 8: Global SSH gradient field reconstruction (August 4, 2013) obtained by OI, (post-)AnDA, VE-DINEOF, FP-ConvAE and FP-GENN for a joint assimilation/learning of along-track nadir with wide-swath SWOT data 